\title{
ANÁLISIS DEL MERCADO DE LAS AEROLÍNEAS TRADICIONALES Y LOW COST EN ECUADOR
}

\author{
Analysis of the Traditional and Low Cost Airline Market in Ecuador
}

\author{
German Jazmany Zambrano Verdesoto, MsC \\ Universidad Estatal de Milagro, Ecuador \\ https://orcid.org/0000-0002-1642-2799 \\ licgerjaz@hotmail.es
}

Palabras claves: Precios, Economía, Servicios, Aerolíneas, Mercado.

Keywords: Prices, Economy, Services, Airlines, Market.
Recibido: 09 de junio de 2020

Aceptado: 16 de octubre de 2020

\section{RESUMEN}

El desarrollo constante que tienen las aerolíneas en Ecuador es importante para incrementar la economía del país, siendo este factor principal para que los clientes tengan una ventaja significativa al momento de comprar un pasaje, esta investigación pretende realizar un estudio de las principales funciones que tiene en el mercado las aerolíneas tradicionales y low cost en Ecuador. El objetivo fundamental es realizar un análisis comparativo de precios y los servicios y cómo se ha desarrollado en el mercado local las aerolíneas tradicionales y low cost. El estudio que se aplicó es de análisis bibliográfico y de las fuentes información que se utilizan para el análisis de precios y servicios son los sitios web que tienen las aerolíneas.

\section{ABSTRACT}

The constant development that airlines have in Ecuador is important to increase the economy of the country, being this main factor so that customers have a significant advantage when buying a ticket, this research intends to conduct a study of the main functions it has in the market for traditional and low cost airlines in Ecuador. The main objective is to carry out a comparative analysis of prices and services and as the traditional and low cost airlines have been developed in the local market, the study that will be applied is bibliographic the sources of information that will be used for the analysis of prices and prices are the websites that airlines have. 


\section{INTRODUCCIÓN}

El desarrollo comercial que tiene las líneas aéreas en todo el mundo hace cada vez aumentar la oferta y demanda de este servicio, unas de las características de estas son las conexiones que son demoradas y las esperas frecuentes en este servicio, por lo cual el cliente opta por otros servicios que pueden brindar las diferentes aerolíneas de bajo costo.

Los segmentos de mercado que tienen las aerolíneas tradicionales se basan en los servicios que pueden ofrecer cada aerolínea, los cuales se basarán por los costos que tienen que pagar los clientes por los servicios que ofrecen, y entre más se incrementan los servicios los valores del pasaje aumentarán. De manera, que el estudio de los precios está dado por tres principales estrategias que se basan en la fijación de precios por el valor para el cliente como las aerolíneas tradicionales que tienen su enfoque en los servicios que ofrece y los precios que están enfocados; las aerolíneas low cost su valor agregado que brindará a sus clientes con pasajes económicos por servicios básicos; la otra estrategia se basa en la fijación de precios basados en el costo, estos precios estarán acorde a la disminución de los servicios que ofrece cada aerolínea, está estrategias al tener una relación vinculante con los clientes por el precio, los clientes obtienen mayores beneficios al comprar un boleto aéreo.

Es objetivo fundamentar y mostrar cómo los elementos del producto han evolucionado en sus diferentes dimensiones, tanto las aerolíneas tradicionales que han implementado diferentes productos basados en un servicio en la cual a los clientes se les brindan mejores alternativas para seleccionar un vuelo, y, las aerolíneas low cost que también han desarrollado diferentes servicios que van desde lo más básico hasta seleccionar con más y mejor calidad el servicio.

\section{DESARROLLO}

En Ecuador operan veinte líneas aéreas de las cuales doce son tradicionales entre ellas están: Aeroméxico, Aereoregional, American Airlines, Air Europa, Iberia, Avianca, Delta, Latam, United, KIm, Copa Airlines, Air Europa, Plus Ultra y la aerolíneas de low cost esta Spirit, Wingo, Laser Airlines, Interjet, Air France, Gol, Jet blue, de la cuales hay aerolíneas que tiene vuelos a Europa como KLM, Air France, Air Europa, Plus Ultra, Latam, Avianca, Iberia que salen de Guayaquil o Quito con destino como España, Italia y Francia incluso una de ella como KLM realiza vuelos Asia y Medio Oriente, las otra como Copa, Latam, Avianca, United, Tame, Interjet, Wingo, Laser Airlines, Gol tiene conexiones en Latinoamérica y Norteamérica como vuelos directos a Panamá de allí a otros países como Argentina, Chile, Perú, Bolivia, Brasil.

El desarrollo de las aerolíneas tradicionales o también denominadas de bandera que nacen como una forma de identificar al país de origen; como, por ejemplo: la línea aérea Tame de Ecuador que inicia su funcionamiento en el de 1962. Serna (2014) describe. Una de las características principales de las compañías tradicionales es el desarrollo de sus operaciones centro-radial, concentrando rutas en un centro o distribuidor (hub) desde donde se conecta con el resto de los destinos por lo que los tiempos de inmovilización de las aeronaves de este tipo de empresas son bastante largos. (p.9)

A pesar de la desventaja que tiene este sistema de aerolínea tradicional su enfoque hacia el cliente es ventajoso; por ejemplo, una de aerolíneas como American Airlines en un vuelo de Ecuador del aeropuerto José Joaquín de Olmedo de Guayaquil New York aeropuerto JKF define la fechas de ida y vuelta y elije el destino una vez dado este resultado, le saldrá la tarifa más baja y flexible, también cabina principal y ejecutiva un pasaje del 01/11/2019 al 28/02/2019 que es por visita a familiar estáa $602 \$$ saliendo en un horario 11:15 pm y llegando el 02/11/2019 en el horario de 7: 29 pm con una duración de 19 h 14m, siendo una buena opción para el cliente.

También, en la misma página puede elegir unos días antes del primero de noviembre o después con otras tarifas similares y también por los horarios que pueden subir el costo del boletó aéreo, en la misma fecha pero en un horario de salida 5: 44 am y llegada 5:29pm con una duración de $10 \mathrm{~h} 13 \mathrm{~m}$ el pasaje costaría $672 \$$ es una diferencia significativa a la hora de seleccionar un vuelo y la diferencia la marcara por el tiempo de espera; después, le da una opción para elegir el asiento en el momento de la reserva, existen asientos que se puede comprar a un valor $79 \$$ que prometen ser de más espacios y preferencias en el embarque, y otro, que le brindan espacio estándar y ubicación conveniente con un valor de 35\$; y otra opción, que no tiene precio y puede elegir los asientos que quedan disponibles, también la reserva dura 24 horas para elegir comprar su pasaje puede pagar con tarjeta de crédito o puede pagar con un recargo de $25 \$$ en las oficina de American Airlines.

En esta misma comparación la aerolínea Latam saliendo del aeropuerto José Joaquín de Olmedo de Guayaquil haca EEUU al aeropuerto JFK con las misma fechas de salida del 01/11/2019 al 28/02/2019 pero con la diferencia que tiene un vuelo directo pero con ciertas similitudes en que puede seleccionar día antes de la fecha propuesta así como también 
días después de la fecha propuesta anteriormente esta aerolínea tenía para sus clientes cabina Economy la cual incluía equipaje de mano de $8 \mathrm{~kg}$ y bolso para llevar un ordenador y 2 maletas de $23 \mathrm{~kg}$ la cual la compra de este viaje era muy beneficioso para un segmento de mercado que viajaba por comprar, en la actualidad tiene tres tarifas en cabina económica que denomina light que permite un equipaje de mano una maleta y cambios por un costo de $300 \$$ y acumula 1567 el costo del pasaje de ida esta $475 \$$.

La tarifa Plus tiene un equipaje mano de $8 \mathrm{~kg}$, dos maletas de 23kg selección de asiento, igual en cambios de fechas de $300 \$$ y el $30 \%$ de devolución ante de los vuelos y también dan 1792 millas y el valor del pasaje es de $526 \$$.

La tarifa Top permite un equipaje de mano de $8 \mathrm{~kg}$, dos maletas de $23 \mathrm{~kg}$, elección de asiento sin costo y asiento LATAM también sin costo, los cambios tampoco tienen costo y la devolución antes de vuelo es del $100 \%$ le dan 2342 millas y el pasaje de es $649 \$$

En esta misma selección el pasaje de regreso es $749 \$$ con la cabina Economy Light y con los mismos atributos del pasaje de ida el pasaje con la tarifa Plus es $799 \$$ y la ta4rifa Top es de $861 \$$.

La tarifa seleccionada es la light que el valor total del pasaje es 1224\$, esta aerolínea también tiene la opción de reserva del pasaje de 24 horas para poder cancelar.

En definitiva, las dos aerolíneas tiene los vuelos con el mismo destino la diferencia se basa en las ventaja que le pueden brindar a sus clientes los que marca la diferencia entre la dos, la primera tiene un vuelo con una escala de tiempo de espera aproximado de 12 horas que el pasaje es $602 \$$, mientras que Latam en un vuelo directo su pasaje es $1224 \$$ en equipaje las dos tienen la misma opción en precio la diferencia es significativa con una diferencia de 622 dólares, American también ofrece un vuelo con escala con un tiempo de duración de 10h13m y con un tiempo de espera $3 \mathrm{~h} 18 \mathrm{~m}$ la cual el pasaje es $672 \$$ con una diferencia del valor que tiene Latam es de $552 \$$, en latam tiene las opciones de cabina básica en la tiene tres tarifas para la elección del viajero puede tomar otra opción de tarifa Plus en la cual puede llevar dos maletas el costo también aumenta.

Las aerolíneas de bajo costo se están posicionando en mercado ecuatoriano con algunas aerolíneas como Wingo que opera de Guayaquil a Bogotá, Spirit opera de Guayaquil a EE. UU, y así mismo, Gol que opera a Brasil, Laser a Caracas, Air frase, de Quito a París, Interjet de Guayaquil a Cancún estas aerolíneas están operando en Ecuador con un crecimiento significativo en el mercado. Casa gualda \& García (2013) define: Una aerolínea de bajo coste o aerolínea low-cost es una aerolínea que generalmente ofrece bajas tarifas a cambio de eliminar muchos de los servicios tradicionales a los pasajeros. El concepto surgió en los Estados Unidos antes de extenderse por Europa a principios de los 90, y de ahí, al resto del mundo. Originalmente, el término era empleado dentro de la industria de la aviación para referirse a compañías con costes de operación bajos o menores que los de la competencia. (p.21)

En Ecuador hay varias líneas aéreas low cost como Wingo, Sprit, Jet Blue las dos últimas tienen los mismos destinos por ejemplo a Spirit tiene frecuencias a Fort Lauderldale EEUU, y así también: Jet Blue en una comparación de precios y de servicios que ofrecen hay diferencias por ejemplo en sitio web de Spirit en un vuelo directo en fechas del $01 / 11 / 2019$ al 09/11/2019 el costo del vuelo en club se tarifas es $338,81 \$$, la cual le permite un asiento aleatorio y un objeto personal, pero este valor es solo si perteneces a ese club pero para tener acceso tiene que pagar $59 \$$ la cual puede acceder a esos precios; pero, también hay la opción de elegir asiento, objeto personal, bolso de mano, bolso a cuadro más acceso directo a embarque y modificar el vuelo a un precio de 521 \$ que vendría a ser un vuelo estándar.

En esta concepción en el sitio web de Jet Blue ofrece vuelos directos de Guayaquil a Fort Lauderdale la cual su mecanismo es distinto no tiene club para ofrecer tarifas bajas, pero al pasajero le permite elegir asientos y llevar un objeto personal el costo del pasaje es de $420 \$$, también tiene otra tarifa blue plus con un valor $485 \$$ de la cual permite llevar un objeto personal y una maleta y también tiene otra tarifa flex azul que permite llevar una maleta adicional a las tarifas anteriores y el valor sube sustancialmente a $666 \$$ las dos aerolíneas se paga inmediata no se permite reservar las 24 horas como las tradicionales.

\section{Estudio de los segmentos de públicos y consumo de las aerolíneas tradicionales y low cost}

Es importante destacar que las aerolíneas tradicionales y low cost desarrollan segmentación para diferenciar a los consumidores y no gastarsus recursos generalizando sus servicios. Monferrer (2013) define. "Segmentar es diferenciar el mercado total de un producto o servicio en grupos diferentes de consumidores, homogéneos entre sí y diferentes a los demás, en cuanto a hábitos, necesidades y gustos, que podrían requerir productos o combinaciones de marketing diferentes" (p.57). 
La diferenciación que realizan las aerolíneas se basan es estudios previos para conocer las necesidades, gusto, preferencias que tienen los clientes para poder aplicar diferentes estrategias de marketing que estén direccionadas a los clientes; por ejemplo, la aerolínea como Latam en sus vuelos de Guayaquil a EEEUU tiene un segmento ya definido se enfoca en clientes que les gusta un viaje directo, y también con status económico alto ya que los pasajes se incrementan el $50 \%$ más de un vuelo que en otra aerolínea.

\section{Incidencia que tienen los productos, precios, servicio y audiencia de las aerolíneas tradicionales y low cost.}

El desarrollo constate que tienen las aerolíneas a nivel mundial permite realizar cambios innovadores en sus productos y servicios que ofrecen a los clientes, en Ecuador hay 19 líneas aéreas entre tradicionales y low cost la cual ofrecen servicios diferenciados que llamen la atención de los consumidores, define. Kotler y Armstrong (2013): "Un producto como algo que puede ser ofrecido a un mercado para su atención, adquisición, uso o consumo, y que podría satisfacer un deseo o una necesidad" (p.196). En el mercado de las aerolíneas ha crecido en los últimos años en Ecuador por el motivo que las autoridades han puesto de manifiesto el cielo abierto la cual las compañías ven como una ventaja este mercado para realizar sus operaciones por ejemplo la aerolínealow cost en la cual le realizan un reportaje. Vilarreal (2019) describe que la aerolínea Plus Ultrainicio sus operaciones a partir del 10 de julio con tres frecuencias semanales en la ruta Madrid-Quito-Guayaquil- Quito- Guayaquil- Madrid, ingresando al mercado a competir con tradicionales como KLM, Latam, Avianca, Air Europa que tiene el mismo destino.

\section{Estrategias de precios planteadas por las aerolíneas tradicionales y low cost}

Las aerolíneas desarrollan diferentes estrategias de precios basadas en la demanda que existe en mercado la participación de la aerolíneas tradicionales se basaban en demanda de clientes que tiene el mercado y el desarrollo de estrategias se basaban según las necesidades del clientes, por ejemplo hace diez años no existían seis aerolíneas para viajar a Europa especialmente a España con mayor apertura al mercado Ecuatoriano estas aerolíneas se ven obligados a realizar estrategias que permitan captar a los clientes con referencia a este mercado según la coordinación Estadísticas del Ministerio de turismo de Ecuador en año 2017 ingresaron 58727 realizaron viajes de Ecuador a España mientras en el 2018 aumento significativamente a 103008 siendo el tercer país en participación en este mercado el primero es EEUU con 351709 y el segundo es Colombia que tienen 323345 con estos datos las aerolíneas ven una gran oportunidad para invertir en Ecuador.

Goñi (2008) define. "La estrategia de precios determina un precio competitivo para un segmento particular del mercado, basado en una estrategia de posicionamiento" (p.20). Las empresas tradicionales tienen una participación que se basa en un segmento de mercado ya definidos basados en: los precios, los destinos geográficos y servicios extras que ofrecen.

Kotler y Armstrong (2013) "Sugiere tres principales estrategias de precios: fijación de precios basada en el valor para el cliente, fijación de precios basada en el costo y precio basado en la competencia" (p.257).

\section{-Fijación de precios basada en el valor para el cliente}

Los desarrollos de estas estrategias en las aerolíneas son fundamentales para captar a los clientes, los costos de los precios deben basarse en las diferentes operaciones que tienen cada aerolínea. por ejemplo un vuelo directo en una aerolínea como Latam de Guayaquil a EEUU el costo según el sitio web de latam en la fecha de 21 de octubre al 31 de octubre el precio es de $922 \$$ con un opción a equipaje de mano, en esta relación la aerolínea de Copa la misma fecha de 21 de octubre al 31 de octubre y el mismo destino el costo del pasaje es de $617 \$$ pero este pasaje es con una escala en Panamá y el tiempo es de 8horas 23minutos y con una ventaja de realizar la reserva de 1 día gratis y si quiere extender 3 días tendría que pagar $34 \$$, la diferencia será dará por lo económico, fiabilidalidad que tiene el cliente, y por los servicios extras que brinde la aerolínea.

\section{-Fijación de precios basada en el costo}

Las aerolíneas tienen diferentes formas de fijar un precio, estos pueden ser dados por los diferentes servicios que puedan tener y la calidad que le pueden hacer percibir al cliente. Lovelock y Wirtz (2009) describe. "Los productos de servicios consisten en un bien fundamental, que responde a una necesidad primaria del cliente, y en un conjunto de elementos del servicio complementario, que ayudan al cliente a utilizar el producto fundamental de manera eficaz, así como a agregar valor por medio de mejoras bien aceptadas" (p.23).

La aerolíneas low cost como Wingo y Spirit llaman la atención por los precios bajos que ofertan en el mercado, esto vendría a ser su principal estrategias de venta y también lo que más cautiva a los clientes al momento de elegir un viaje, mientras que las aerolíneas tradicionales ofrecen sus vuelos con diferentes servicios como cabina principal con 
tarifa más baja, el valor de pasaje de Ecuador a EEUU está en los $6333 \$$ y también ofrece tarifa flexible que es para la clase ejecutiva está en $2300 \$$ que tiene servicios adicionales como asientos cómodos, internet, cambio de fechas del vuelo sin recargo y maletas adicionales su enfoque está en generar valor en los servicios que ofrece en cada tarifa.

\section{-Fijación de precios basado en la competencia}

Con el desarrollo comercial que tienen las aerolíneas en Latinoamérica cada vez se introducen a nuevos mercados como en Ecuador teniendo dos principales aeropuertos y operan un aproximado de veinte aerolíneas en que tienen diferentes destinos geográficamente bien definos entre los que destacan: Europa, Asia, EEUU, Sudamérica. Goñi (2008) expresa: "Cuanta más competencia hay en el mercado, la posibilidad de fijar los precios de los productos unilateralmente es mínima o nula, de ahí que las empresas siempre están pendientes del que hacer de su competencia" (p.18).

Los precios se fijarán dependiendo la oferta y demanda cuando mayor sea la oferta los precios de un producto o servicio van a estar fijados por debajo de lo normal y mientras la demanda tenga las necesidades la oferta establecerán sus precios de acuerdo a los servicios que promocionarán; por ejemplo, en las aerolíneas tradicionales sus precios se fijan por sus servicios que ofrecen si un pasajero quiere cabina económica el precios de esa tarifa va a ser inferior al de la clase ejecutiva, también puede basarse en si en vuelo directo o con escala, en directo tendrá mayor valor. En otro contexto, en las aerolíneas low cost sus precios están fijados por su promesa de viajes, a diferencia de las aerolíneas tradicionales.

\section{Evolución que tienen los elementos de producto en las diferentes dimensiones de productos en las aerolíneas tradicionales y low cost.}

Según Monferrer (2013) describe tres dimensiones que componen el todo del producto:

a) El producto básico: en las aerolíneas low cost por ejemplo los clientes se basan su viaje en la parte económica lo ven como prioridad al momento de seleccionar una aerolínea, en cambio en las aerolíneas tradicionales los clientes se enfocan en los servicios que pueda ofrecer la aerolínea.

b) El producto real: es producto parte de lo básico hasta llegar al producto real donde los servicios que puedan ofrecen las aerolíneas tiene como su mayor prioridad por ejemplo las aerolíneas tradicionales tienen mayor cantidad de servicios al momento de comprar un boleto aéreo para su clientes pero los valores dependerán de las necesidades y prioridades que tengan para su viaje, en el mismo sentido las aerolíneas low cost tienen como prioridad su valor agregado en el precio a bajo costo, pero en también ofrece más servicios pero el incremento del valor aumenta y la calidad al momento de seleccionar una marca se basara en la experiencia que haya tenido el cliente.

c) Producto aumentado: el desarrollo de este producto será dado por servicios adicionales que ofrece el producto real, basándose en la aerolíneas tradicionales como Latam ofrecen en su tarifica económica que son: light, plus, top en la cual los precios varían según la necesidad del cliente y en cabina premiun business tiene una tarifa la top la el pasaje es superior y puede tener capacidad de equipaje, puede devolver el $100 \%$ de tarifa antes de vuelo, pueden financiar su pago, y también ofrecer garantías en el equipaje.

\section{CONCLUSIÓN}

Las aerolíneas tradicionales o low cost tiene sus ventajas hacia el cliente ya que permiten tener opciones para comprar un boletó aéreo, tal es el caso de American que permite la selección de asientos, Latam ofrece su vuelo directo a mayor precio con una diferencia significativa de hasta $50 \%$ a la hora de comprar un vuelo.

Las opciones son variadas para los clientes que a la hora de elegir se basan en las necesidades que tengan al momento de viajar, los servicios que ofrecen cada aerolínea marca la diferencia en los precios, estos precios se basan en los recursos que cada cliente tiene para elegir un vuelo directo, si así lo desea, pero si no poseen el poder adquisitivo tendrán suficientes opciones para comprar un vuelo.

En Ecuador en los últimos años han llegado más aerolíneas de bajo costo como Spirit, Jet Blue, Wingo, esta última realiza vuelos de Guayaquil a Bogotá a precios económicos y se ha constituido en una opción para viajar a distintas partes del mundo, a pesar de que modelo de negocio ha surgido en EEUU y Europa en Latinoamérica ha ganado mercado captando nuevos clientes. 
La introducción de este modelo de viajes económicos ha hecho que las aerolíneas tradicionales también modifiquen su estructura y creen vuelos a bajo costo implementando estrategias como la que realiza Latam en la cabina económica con tres tarifas que son light, top y plus que le da al pasajero diferentes opciones para comprar su boleto.

\section{BIBLIOGRAFÍA}

1. Casagualda, M., \& Garcia , A. (2013). Son la low cost menos seguras que las aerolías tradiconales. España: Universidad Autonoma de Barcelona . Obtenido de https://ddd.uab.cat/pub/tfg/2013/113514/CasagualdaMarcGarciaAlexisTFGAa2012-13.pdf

2. Goñi, N. (2008). El precio. Variable clave en el Marketing. México: Pearson Educación. Obtenido de https://utecno.files.wordpress.com/2013/09/el-precio.pdf

3. Kotler , P., \& Armstrong, G. (2013). Fundamentos de Marketing. México: Pearson Educación.

4. Lovelock, C., \& Wirtz, J. (2009). Marketing de Servicios perosnal, tecnología y estrategia. México: Perarson Educación. Obtenido de https://decisiondelconsumidor.files.wordpress.com/2017/07/marketing-de-servicioschristopher-lovelock.pdf

5. Monferrer , D. (2013). Fundamentos de Marketing. España: Publicacions de la Universitat Jaume I. Obtenido de http://repositori.uji.es/xmlui/bitstream/handle/10234/49394/s74.pdf

6. Vilarreal, P. (1 de Febrero de 2019). La aerolínea low cost Plus Ultra operará la ruta Madrid - Quito - Guayaquil desde julio. El Universo . Obtenido de https://www.eluniverso.com/noticias/2019/01/21/nota/7151261/plusultra-aerolinea-low-cost-operara-vuelos-ecuador-espana-julio

7. American Airlines ( 28 de octubre de 2019) Reserve. Recuperado de https://www.aa.com/homePage.do?locale=es_EC

8. Copa Airlines $(20$ de septiembre de 2019) Reserve su Viaje. Recuperado de https://www.copaair.com/es/web/gs/home

9. Despegar.com (22 de septiembre de 2019) Reserva tu vuelo. Recuperado de https://www.despegar.com.ec/

10. González, P. (18 de enero de 2018) Más alternativas se abren en 2019 para los viajeros aéreos de Ecuador. El Universo. Recuperado. https://www.elcomercio.com/actualidad/aerolineas-vuelos-ecuador-conectividadpasajeros.html.

11. La aerolínea española Plus Ultra inicia operaciones en Ecuador (12 de julio de 2019) El Telégrafo. Recuperado de https://www.eltelegrafo.com.ec/noticias/economia/4/plusultra-operaciones-ecuador

12. Larenas, N. ( 01 de septiembre de 2019) Las estadísticas de turistas extranjeros en Ecuador 2018. [Mensaje en un blog] Recuperado de https://www.nlarenas.com/2019/01/estadisticas-de-turistas-extranjeros-enecuador-2018/

13. Latam Airlines (30 de octubre de 2019) Reserva tu vuelo. Recuperado de https://www.latam.com/es_ec/

14. Larenas, N. (01 de octubre de 2019) Interjet inició vuelos a Ecuador volando hacia Guayaquil [Mensaje en un blog] Recuperado de https://www.nlarenas.com/2019/10/interjet-inicio-vuelos-a-ecuador-volando-haciaguayaquil/ 